\title{
UNA REVISIÓN DE LA TEORÍA DE ÁREAS MONETARIAS ÓPTIMAS
}

\author{
Lic. Virginia Corbella*
}

enviado: octubre 2014 - aceptado: abril 2015

\section{INTRODUCCIÓN}

Es frecuente en la literatura técnica encontrar dos enfoques para la liberalización del comercio internacional: el enfoque regional y el enfoque internacional. Dentro de este último se encuentran las ideas propuestas por el Acuerdo General sobre Aranceles Aduaneros y Comercio (GATT), cuyo objetivo es reducir las barreras arancelarias y otros obstáculos al comercio y eliminar todo trato discriminatorio en materia de comercio internacional. Los avances más importantes alcanzados en la liberalización del comercio internacional durante los años ' 50 y ' 60 se han realizado mediante una serie de negociaciones comerciales multilaterales o rondas auspiciadas por el GATT. Incluso, algunos economistas llegaron a la conclusión de que el GATT contribuyó en esos años al crecimiento mundial gracias a las continuas reducciones de aranceles.

En cambio, el enfoque regional comprende los acuerdos entre un conjunto de países cuyo propósito es establecer el libre comercio entre ellos, manteniendo barreras comerciales con el resto del mundo. A estas negociaciones se las suele llamar acuerdos de comercio preferencial. Ejemplos de ellos son la Unión Europea, Mercado Común del Sur, Comunidad Andina, entre otros. Los mismos pueden optar por diferentes formas de integración. La integración económica es un proceso mediante el cual dos o más países eliminan barreras comerciales facilitando el intercambio entre ellos. Existen diferentes grados de integración. La misma puede referirse tanto al libre intercambio de bienes y servicios entre las naciones socias,

* Instituto de Investigaciones Económicas y Sociales del Sur (IIESS) - Departamento de Economía Universidad Nacional del Sur. E-Mail: corbella.virginia@uns.edu.ar 
como también puede implicar el libre flujo de factores productivos, la coordinación macroeconómica y la unión monetaria. Balassa (1961) realizó una clasificación entre las distintas etapas del proceso de integración. Las mismas consisten en:

- Zona de libre cambio: es un área formada por más de un país en la cual se eliminan los aranceles aduaneros y demás barreras comerciales entre ellos. - Unión aduanera: es un área de libre comercio a la cual se le agrega el establecimiento de un arancel externo común para con los terceros países no socios.

- Mercado común: es una unión aduanera en la cual se libera la circulación de los factores productivos, considerando como tales al capital y el trabajo. - Unión económica: añade a la forma anterior cierto grado de armonización de políticas internas y la cesión parcial de soberanía por partes de los países a órganos de jerarquía supranacional.

- Unión total: es la etapa más profunda de integración, unificándose las políticas coyunturales, sociales y fiscales y procediéndose a la unión monetaria. Ejemplo de este caso es la Unión Europea.

Las formas más básicas, como las zonas de libre comercio y las uniones aduaneras, se refieren esencialmente a los aspectos comerciales. Mientras que la forma de integración que excede ese aspecto es la unión económica. Esta se da cuando dos o más países se unen, creando un mercado común, en donde se han eliminado los aranceles de importación y se ha establecido un arancel externo común junto con la libre circulación de todos los factores de producción, procediendo finalmente a unificar sus políticas fiscales, monetarias y socioeconómicas. Una unión económica es la forma más completa de integración. Algunos de los ejemplos más citados en la teoría de la misma son la Unión Europea y los Estados Unidos ${ }^{1}$.

¿Por qué se comienza en la literatura a hablar de zonas monetarias óptimas? La teoría sobre las áreas monetarias óptimas comienza a formarse en la década de los 60 y está relacionada con las propuestas de un grupo de economistas de corte liberal que habían comenzado a estudiar los tipos de cambio como herramienta para conservar la estabilidad económica. Entre dichos economistas se destacarían Meade (1951), Friedman (1953) y Scitovsky (1958); quienes apoyaban un régimen de tipos de cambio flexibles entre áreas monetarias para facilitar el ajuste y alcanzar el equilibrio externo. Mundell (1961) se verá inspirado por estos autores

1 Se considera a Estados Unidos como una unión de estados provincia que han alcanzado el máximo nivel de integración. 
para establecer las bases de la teoría de las áreas monetarias óptimas (Martínez Romera, 2013; Manchón, 2004).

Una unión económica y monetaria se puede interpretar como una integración a nivel macroeconómico de los países, en donde sólo quedaría por delante, en teoría, la Unión Política, también denominada en la literatura zona u área monetaria óptima (AMO). Este concepto desarrollado por Mundell (1961), es de uso general en la literatura técnica y se entiende que la AMO:

(...) se da en los casos en que los beneficios de renunciar al uso del tipo de cambio como mecanismo interno de ajuste son superiores a los costos de adoptar una moneda única en un sistema de cambio fijo. La utilidad/sustentabilidad de una AMO a menudo se determina en función de la movilidad de la fuerza de trabajo, el tamaño y la apertura de la economía, la semejanza de la estructura productiva y la incidencia (o asimetría) de las perturbaciones económicas. Si hay una marcada relación comercial mutua, peligro de shocks externos y sincronización de los ciclos económicos, aumentan los beneficios netos que se espera obtener de la adopción de una moneda y de una política monetaria común. A decir verdad, cuando los shocks afectan de manera similar y en el mismo punto del ciclo económico a todos los países no es necesario modificar el tipo de cambio (Escaith, 2003: 59).

Avanzar en la integración requiere afrontar otros aspectos además de los comerciales. Entre los mismos se encuentra la coordinación de las políticas monetarias, cambiarias, financieras, presupuestarias y fiscales, cuya determinación es cedida en cierto grado a los organismo supranacionales que van a ser la autoridad máxima del área integrada. En palabras de Tugores, "una unión monetaria conlleva elementos de corte político ligados a la soberanía que la convierten en un tema delicado [...] el respaldo político fue vital para estos procesos de integración" (Tugores, 1997:185).

Por ejemplo, cuando un país se enfrenta a una crisis, generalmente cuenta con herramientas para poder afectar el tipo de cambio y así mejorar la competitividad internacional de sus productos generando una devaluación del mismo. Si el país pertenece a una unión, verá limitado su campo de acción porque su tipo de cambio ahora permanece fijado permanentemente y es el mismo para todos los Estados miembros. Sólo es modificado por decisión de las autoridades supranacionales que tienen a su cargo la política monetaria y cambiaria de la unión monetaria. Esta situación se ha podido apreciar durante la última crisis sufrida por países miembros de la Unión Europea. 
En este contexto, esto es interpretado muchas veces como una limitación a la autonomía nacional para responder ante shocks externos que afecten la economía doméstica del país miembro. En otras palabras, se lo considera como un costo al momento de analizar la conveniencia de formar parte o no de una unión monetaria.

Pero una integración de este nivel también ofrece beneficios, como por ejemplo, que al adoptar una moneda única las transacciones que antes eran internacionales y debían superar "fronteras" ahora están expresadas en una misma moneda y se mueven dentro de un solo territorio, la zona monetaria. Esto provoca que se reduzcan los costos de incertidumbre cambiaria y de transacción, dado que en ese contexto se expresa todo en una misma moneda, generando recursos que pueden ser utilizados más eficientemente.

De la Cuba y Winkelried (2004) sintetizan los beneficios y costos de pertenecer a una AMO en los siguientes términos:

Un beneficio importante radica en la eliminación de la conversión de monedas, actividad que entorpece y encarece los flujos comerciales y financieros; asimismo, una moneda común evita la incertidumbre en las transacciones internacionales asociada a la volatilidad cambiaria. (...) Por el lado de los costos, la moneda única supone que cada país renuncia a la posibilidad de adoptar una política monetaria autónoma al ceder tal facultad a una institución supranacional. Ello puede ser costoso si es que los miembros de la unión monetaria enfrentan ciclos económicos diferentes (De la Cuba y Winkelried, 2004: 3).

El trabajo se estructura de la siguiente manera: en la segunda sección se describen brevemente los criterios tradicionales para un área monetaria óptima. En la tercera parte se detalla el análisis costo-beneficio que los países deben realizar a la hora de evaluar su incorporación a una zona monetaria óptima. A través de un modelo sencillo se muestra cómo es el funcionamiento de la toma de decisión. En la cuarta sección se presenta la endogeneización que se ha producido a lo largo del tiempo sobre los criterios tradicionales, y los cambios que esto produce al momento de evaluar si el país cumple con los requisitos establecidos para su ingreso a la AMO. En la última sección se realizan algunas consideraciones, a partir de las diferencias encontradas entre los enfoques AMO tradicional y nuevo y la posibilidad de que exista una AMO para América Latina. 


\section{CRITERIOS TRADICIONALES PARA UN AMO}

Existen una serie de criterios, considerados tradicionales, que permiten configurar un área monetaria óptima, los cuales son:

a) Siguiendo el criterio de McKinnon (1963), debe existir una elevada apertura o grado de comercio entre las partes que están evaluando formar parte del territorio de un área monetaria óptima, para que ésta pueda superar de la mejor manera posible un shock asimétrico o la situación en la cual los países miembros se encuentran en etapas distintas del ciclo económico. El grado de apertura exterior de la economía lo mide a través del cociente entre los bienes comercializables internacionalmente y los no comercializables.

Es importante expresar que la principal limitación de este criterio es el supuesto básico en el que basa su modelo. McKinnon (op.cit.) plantea una situación idónea en la que los precios en moneda extranjera se mantienen fijos, esto significa que existe estabilidad de precios en el resto del mundo. Sin embargo, si un país tiene establecido un tipo de cambio fijo con otros países y se produce una perturbación fuera de sus fronteras, parte de los efectos de dicha perturbación afectarán su economía. En consecuencia, hay que contemplar en los resultados posibles la posibilidad de que un país se vea afectado por shocks producidos en el resto de los países con los que forma un área monetaria óptima, con lo que se ve opacada la idoneidad del sistema de tipo de cambio fijo.

b) Siguiendo el criterio de Mundell (1961), entre los Estados en cuestión debe presentarse una elevada movilidad de factores capaz de responder a perturbaciones asimétricas entre partes del territorio. Una de las principales observaciones que se le hacen al análisis de Mundell, y que es señalado por Fleming (1971), es que este autor habla genéricamente de movimientos de factores. Se refiere indistintamente al trabajo y al capital al hablar de movimiento de los factores, aunque en su análisis hace referencia únicamente al factor trabajo.

Es fácil comprender cómo la movilidad del trabajo ayuda a reducir los desajustes producidos por un shock $\mathrm{y}$, de este modo, disminuyen los costos de formar un área monetaria. Sin embargo, no es tan evidente la contribución para disminuir los efectos negativos de una perturbación y los resultados que produce la movilidad del capital. Su efecto es ambiguo y puede llegar incluso a ser contraproducente. Es necesario conocer 
la naturaleza del desequilibrio. Por ejemplo, si se considera un shock que afecta directamente a la balanza por cuenta corriente, el efecto del movimiento de capital es ambiguo, pero es perjudicial si se considera una perturbación que provoque un cambio exógeno en el incentivo a invertir en alguno de los países miembros con respecto al resto de naciones que componen la AMO.

McKinnon (op.cit.) aclara con respecto a este criterio, sin poner en duda su importancia, que el grado de movimiento hay que tomarlo en consideración una vez formada el área monetaria, dado que su formación afecta a la movilidad del trabajo y del capital. Dicho en otras palabras, como lo afirman Frenkel y Rose (1998), la movilidad de factores es endógena al proceso de constitución de la unión monetaria.

c) Siguiendo el criterio de Kenen (1969), entre las partes que están evaluando formar parte del territorio de un área monetaria óptima debe existir un elevado grado de diversificación de productos, que minimice la posibilidad de shocks asimétricos. De este modo, ante un eventual shock asimétrico, el país que posee diversificada su producción podrá compensar el shock que enfrente un sector de sus exportaciones, con la mejora que puede producirse en otro sector de manera simultánea. Consecuentemente las exportaciones totales se mantendrán aproximadamente estables, o más estables que en una economía menos diversificada, y no requerirá ajustes frecuentes en su tipo de cambio para mantener el equilibrio externo. Otra posibilidad para enfrentar un shock asimétrico es la existencia de un poder fiscal centralizado, capaz de realizar de transferencias de recursos desde los Estados miembros más fuertes económicamente hacia los que se ven afectados por los shocks o costes de ajustes por iniciar el camino para pertenecer a la AMO. Este autor presentó un modelo formal para reafirmar sus conclusiones. Sin embargo, el mismo se basa en supuestos extremadamente fuertes, y si son relajados es imposible comprobar, al menos formalmente, sus proposiciones. ${ }^{2}$

Kenen pone mucho énfasis en el papel desempeñado por la política de cada país y además, sostiene, basándose en cuestiones de política económica, que los países con menor grado de desarrollo no deben formar áreas monetarias, no así los desarrollados. La veracidad de esta afirmación es

2 Kenen supone que la oferta de trabajo es infinitamente elástica con respecto a una tasa de salario nominal dada, y que ésta y el precio internacional del bien importado cambian en la misma proporción. 
muy discutible, ya que los países con menor desarrollo económico podrían obtener de la unión monetaria con otros países, importantes beneficios, como por ejemplo, credibilidad de la política económica, estabilidad de precios y mercados más amplios para sus productos.

Además de los criterios ya analizados y que se pueden considerar fundamentales o tradicionales, se han desarrollado otros que intentan, igualmente, definir las condiciones o características que debe presentar una AMO para que sea una alternativa eficaz:-

a) Integración financiera. Hasta el momento los criterios expuestos toman en consideración las características reales de la economía y atribuyen poca importancia al papel que juega el dinero en la economía. Sin embargo, según Ingram (1973), la característica fundamental de un área monetaria óptima se centra en los aspectos financieros más que en los reales. Coincidiendo con Scitovsky (1958) en que el criterio para delimitar un área monetaria óptima es la existencia de un elevado grado de integración financiera, tanto a corto como a largo plazo, para que los países puedan hacer frente a los shocks externos. Esto significa que, si la integración en el mercado de capital es elevada, los tipos de cambio fijo serán los adecuados para corregir los desequilibrios en los pagos. En vez de ajustarse el tipo de cambio se va a ver modificada la tasa de interés, que producirá un desplazamiento del capital en cantidad suficiente para equilibrar la balanza de pagos. Para que este criterio funcione correctamente, es necesario que la población tenga la convicción de que los tipos de cambio no van a verse alterados. Asimismo, Ingram afirma que la política monetaria debe desarrollarse en conjunto por todos los países que componen el área, mientras que la política fiscal debe ser independiente y tratar de dirigir la inversión hacia zonas deprimidas con el objetivo de estimular la demanda agregada y reducir el desempleo.

b) Integración fiscal. En este caso, una coordinación entre las políticas fiscales minimiza la posibilidad de shocks asimétricos y ayuda a reducir los costos de perder el instrumento del tipo de cambio como mecanismo de ajuste. La existencia de este poder fiscal centralizado mitiga los efectos adversos de una perturbación por medio de transferencias de recursos desde los Estados miembros más fuertes económicamente hacia los que se ven afectados por los shocks o costes de ajustes por iniciar el camino para pertenecer al área monetaria. En este caso las políticas sociales y de bienestar recaerían bajo la autoridad de cada país, no de la autoridad central (Robson, 1998). MacDougall (1975) y Allen $(1982,1983)$ hacen 
hincapié en la importancia crucial de un alto grado de integración fiscal, que maximizaría la viabilidad y la eficacia de las transferencias fiscales dentro de la unión monetaria para facilitar el proceso de estabilización.

c) El grado de integración en el mercado de bienes que tienen los países que quieren formar parte de un proceso de integración. Dicho criterio afirma que, para que un área presente características que puedan ofrecer una alternativa a la política cambiaria a la hora de alcanzar el equilibrio externo e interno, hay que observar el nivel de integración en el mercado de bienes. Una unión monetaria es una de las formas posibles que puede adoptar la integración regional. Normalmente en la bibliografía se encuentra, que la unión monetaria se encuentra sólo en combinación con una unión aduanera o un mercado común, y se sugiere que la unión monetaria debe seguir y no preceder a la institución de los arreglos para la integración de los mercados. Este criterio tiene un nexo conector con el propuesto por Kenen (1969), ya que a mayor diversificación de la estructura de producción más probable será que la integración en el mercado de bienes sea elevada. También puede suceder que este criterio se halle contenido en cierto grado en la propuesta de McKinnon (1963), aunque él se refiere a un grado de apertura comercial medido como ratio entre el comercio y la producción doméstica de bienes previo a la reducción de barreras arancelarias que podrían modificar dicha apertura del país (CEC, 1992a; Robson, 2002).

Los criterios desarrollados hasta el momento, si bien intentan dar ciertas pautas para delimitar una $\mathrm{AMO}$, evalúan una sola característica de la economía y son independientes entre sí. Como son excluyentes, de considerarlos en conjunto, se llega a un resultado incongruente, no pudiendo alcanzar una conclusión taxativa de ellos.

Un ejemplo esclarecedor de esta situación es analizar de forma conjunta a los criterios de integración fiscal e integración financiera, ya que entran en clara contradicción si se recuerda lo anteriormente dicho para cada uno. La integración financiera implica coordinación monetaria pero independencia fiscal $\mathrm{y}$, por otro lado, la integración fiscal implica únicamente la coordinación entre los países miembros de sus políticas fiscales. Este ejemplo pone de manifiesto que dependiendo del criterio en el que basemos el análisis, las recomendaciones pueden ser radicalmente opuestas.

Hay que señalar también que el problema más grave que se puede llegar a dar es que los criterios, además de llegar a conclusiones opuestas acerca de la conveniencia o no de llevar a cabo un proceso de integración, lleven, a veces, a 
resultados que son inconsistentes. Por ejemplo, siguiendo a McKinnon (1963), los países o áreas con economías pequeñas son los que presentan mayor grado de apertura con el exterior, por lo tanto deberían formar un área monetaria; sin embargo, es posible que tales economías no posean una variada estructura productiva, por lo que, de acuerdo con Kenen, deberían mantener un tipo de cambio flexible entre sus monedas. De igual modo, si la economía de un país tiene un grado elevado de movilidad de factores podría producirse un incremento en su especialización productiva, nos encontramos entonces, con una paradoja entre el criterio de Mundell y Kenen. Una condición que indica favorable formar un área monetaria óptima desvirtúa otra condición que indicaba lo mismo.

\section{ANÁLISIS COSTO - BENEFICIO DE PERTENECER A UNA AMO}

Otra forma de plantear la decisión de un país de unirse a un área de tipos de cambio fijo es mediante un análisis de los costos y beneficios económicos de llevar a cabo dicha unión. A continuación se expondrá por medio de un modelo muy simple presentado por Krugman y Obstfeld (2003), cómo estos costos y beneficios dependen, siguiendo el criterio de Mundell y el de McKinnon, de cuán integrada esté su economía con la de sus países socios potenciales.

Ambos criterios predicen, como se detalló más arriba, que los tipos de cambio fijos son más adecuados para áreas estrechamente integradas a través del comercio internacional o con un elevado grado de apertura al comercio exterior y movilidad de factores.

Un país puede conseguir suavizar el impacto de diversos shocks económicos alterando su tipo de cambio. Pero es importante recordar que la flexibilidad del tipo de cambio puede tener efectos potencialmente perjudiciales, por ejemplo, hacer menos predecible los precios relativos o debilitar la decisión del gobierno de controlar la inflación.

Para poder llevar a cabo el análisis costo-beneficio, se necesita un marco en torno a herramientas de ajuste (estabilizadores) que un país sacrifica y las ganancias de eficiencia y credibilidad que puede conseguir por unirse a un área monetaria y fijar su tipo de cambio. "Uno de los principales beneficios económicos de los tipos de cambio fijos es que simplifican los cálculos económicos y proporcionan una base más predecible para las decisiones que implican transacciones económicas (...)" (Krugman y Obstfeld, 2003:640). 
La ganancia de eficiencia monetaria de unirse al sistema de tipo de cambio fijo es igual a los ahorros del país que se une, derivados de evitar la incertidumbre, confusión y los costos de cálculo y transacción que surgen cuando los tipos de cambio fluctúan. En la práctica, es difícil asignar una cifra exacta a la ganancia de eficiencia monetaria. Sin embargo, se puede afirmar que esta ganancia será mayor si el potencial socio comercia intensamente con los países que pertenecen a la unión monetaria o si el comercio entre ellos es amplio. También será mayor si los factores de producción pueden desplazarse libremente entre el país decisor y los países que forman la AMO (Krugman y Obstfeld, 2003)

Seguidamente se presentaran las dos curvas que forman parte del modelo de análisis por parte del potencial nuevo socio del área monetaria y lo que representa cada uno. Luego se mostrará la resolución del mismo, es decir, cómo se llega al equilibrio que permite tomar la decisión de unirse o no a un área monetaria. En el eje horizontal se mide la cuantía en que el país que se une al área está económicamente integrado en los mercados de productos y factores de la AMO. El eje vertical mide la ganancia de eficiencia monetaria para el país de unirse a la AMO.

El primero de los dos elementos a describir que componen el modelo es una curva denominada GG, la cual muestra cómo la ganancia potencial del país decisor de unirse a la zona monetaria depende de los vínculos comerciales de este estado con dicha área. La curva tiene pendiente positiva, la cual muestra que la ganancia monetaria de unirse a un área de tipo de cambio fijo aumenta a medida que la integración económica del país con el área aumenta.

Figura 1. Beneficios y pérdidas de formar una AMO

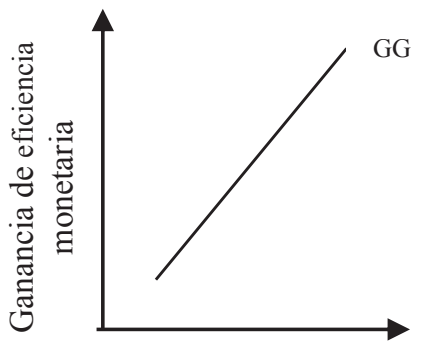

Grado de integración económica

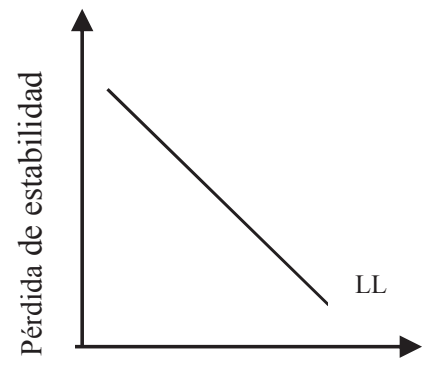

Grado de Integración económica

Fuente: Elaboración propia. 
Este modelo presenta un supuesto implícito importante que es el siguiente: los países que ya conforman un área con tipo de cambio fijo poseen un nivel de precios estable y predecible. Si no lo tuvieran, la mayor variabilidad en el nivel de precios que sufriría el país que está evaluando unirse al área integrada, contrarrestaría probablemente a cualquier ganancia de eficiencia monetaria que un tipo de cambio fijo podría proporcionarle.

Esta aclaración permite considerar como otro beneficio potencial de unirse al área monetaria óptima, a la importancia de la política y credibilidad anti inflacionista de las autoridades monetarias del área hacia el futuro socio. Cuando uno de los países goza de estabilidad de precios, es más probable que el otro logre una baja inflación. Esto se debe a que una integración económica más estrecha conduce a una convergencia internacional de precios y de esta forma se atenúa el margen para una variación independiente en el nivel de precios del país que se une.

Ahora bien, así como existen beneficios también existen costos de pertenecer a un área con tipos de cambio fijos, incluso cuando el área tiene baja inflación. Estos costos surgen debido a que el país que se une al área monetaria renuncia a su capacidad de utilizar el tipo de cambio y la política monetaria con el objetivo de estabilizar la producción y el empleo. Esta pérdida de estabilidad económica está relacionada con la integración económica del país con sus socios de tipo de cambio fijo, la cual se muestra en la segunda curva (LL) del modelo.

En el eje horizontal se mide la integración económica de la nación que se une con el área de tipo de cambio fijo. En el eje vertical se mide la pérdida de estabilidad económica del país por no poder usar a la política monetaria como herramienta de ajuste. La pendiente negativa de la curva LL muestra que la pérdida de estabilidad económica resultante de unirse a un área de tipo de cambio fijo disminuye a medida que la integración económica del país con el área aumenta.

Si la economía de un país se ve perturbada por un cambio en el mercado de productos, es decir, un desplazamiento en la curva de demanda, un tipo de cambio flexible presenta la ventaja de que permitiría atenuar automáticamente el impacto sobre el empleo y la producción, al contrario de un tipo de cambio fijo. En este último caso, al carecer la política monetaria de poder para afectar el nivel de producción y empleo, los efectos económicos son más severos que en una economía con tipo de cambio flexible. La inestabilidad adicional originada por el tipo de cambio fijo es la pérdida de estabilidad económica. 
La magnitud de esta pérdida de estabilidad económica está relacionada con el nivel de integración económica del país con los países que forman la unión monetaria. Si se produce una caída en la demanda agregada de la producción del futuro socio y simultáneamente las demandas agregadas de la unión también se ven desplazadas hacia la izquierda, todas las monedas del área monetaria o la moneda de uso común que poseen, se depreciarán conjuntamente frente a las monedas exteriores, produciéndose una estabilización automática. El problema se presenta cuando únicamente uno de los países socios pertenecientes a la unión enfrenta un shock asimétrico.

Ahora bien, la severidad de la recesión va a depender de forma negativa de la integración económica entre la economía del país que se une y la de los países de la zona monetaria. Es decir, una mayor integración implica una menor recesión $\mathrm{y}$, por lo tanto, un ajuste menos costoso ante un shock asimétrico. Esto se debe a dos motivos. Por un lado, si existen estrechos vínculos comerciales con el área monetaria, una reducción en los precios del país que sufrió la caída de la demanda agregada, conducirá a un aumento grande en la demanda de sus bienes en relación a su producción. De esta manera, el pleno empleo se puede restablecer más rápido. Por otro lado, si los mercados de trabajo y capital están estrechamente vinculados, los trabajadores desempleados pueden movilizarse hacia otros países para encontrar trabajo y el capital puede ser desplazado a usos más rentables en el extranjero. La movilidad de factores y los estrechos vínculos comerciales reducen la severidad del desempleo y la caída en los precios, salarios y tasas de rendimiento.

A continuación se integran las dos curvas para determinar el nivel mínimo de integración económica a partir del cual un país deseará vincularse a un área de tipo de cambio fijo.

El país decisor debería unirse a la unión monetaria si el grado de integración económica entre sus mercados y los de la unión es al menos igual a $\Theta_{1}$, el nivel de integración determinado por la yuxtaposición de ambas curvas. Por debajo de ese nivel no le conviene unirse porque la curva GG se sitúa por debajo de la curva LL. De este modo, la pérdida que sufriría el país por la mayor inestabilidad de la producción y el empleo tras unirse, excede a la ganancia de eficiencia monetaria.

Sin embargo, si el grado de integración económica es $\Theta_{1}$ o mayor, la curva GG se sitúa por encima de la curva LL. En este caso la ganancia de eficiencia monetaria es mayor que el sacrificio de estabilidad económica, y unirse le otorga al país decisor una ganancia neta. 
Figura 2. Ganancia neta de formar una AMO

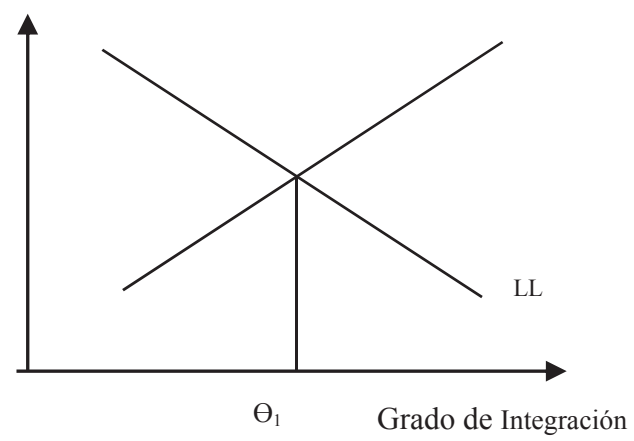

Fuente: Elaboración propia.

El enfoque GG-LL tiene importantes implicaciones acerca de cómo cambios o perturbaciones en el entorno económico de un país afectan la decisión de vincular su moneda con un área monetaria óptima. Considérese, por ejemplo, un aumento en las perturbaciones de la demanda agregada.

Esta situación desplaza la curva LL hacia la derecha. La inestabilidad adicional que sufre el país en la producción y el empleo al fijar su tipo de cambio, para cualquier nivel de integración económica a partir del cual es conveniente unirse, aumenta. La mayor variabilidad en sus mercados hace que el país esté menos dispuesto a entrar en áreas de tipos de cambio fijos.

El modelo GG-LL que se ha desarrollado sugiere una teoría del área monetaria óptima. Dichas áreas son grupos de regiones con economías estrechamente vinculadas por el comercio de bienes y servicios y por la gran movilidad de factores. Esta conclusión se desprende de haber encontrado que un área de tipo de cambio fijo servirá mejor a los intereses económicos de cada uno de los estados miembros si el grado de integración comercial y de mercados de factores entre las economías participantes es elevado.

Si bien el modelo GG-LL es útil para organizar el análisis sobre áreas monetarias, hay que recordar que el modelo se basa en dos de los criterios analizados. $\mathrm{Al}$ menos otros dos pueden afectar los movimientos de curvas y las conclusiones a las que se llega: similitud de estructura económica e integración fiscal ${ }^{3}$.

3 A mayor similitud de estructura económica, mayor es la posibilidad de que se produzca movilidad de los 
El modelo determina que un amplio comercio con el área monetaria facilita que un miembro se ajuste a las perturbaciones que le afecten de forma diferente a él y a sus socios. Pero no especifica qué factores reducirán la frecuencia y magnitud de las perturbaciones específicas de un miembro. Este aspecto es tenido en cuenta por Frankel y Rose (1998), quienes tratarán de identificar esos factores y abordarán el tema de forma endógena en relación a los criterios para delimitar una AMO.

\section{ENDOGENEIZACIÓN DE LOS CRITERIOS PARA FORMAR UNA AMO}

A partir de la experiencia europea, y del trabajo pionero de Frenkel y Rose (1998) donde proponen abordar los criterios que delimitan una zona monetaria óptima de forma endógena, algunos autores se han adherido a este nuevo enfoque y ha surgido una corriente revisionista en la literatura de las áreas monetarias óptimas.

Estos autores sostienen que un país entra a una unión monetaria según ciertos criterios, particularmente, la intensidad de comercio con países miembros de la unión y el grado de correlación de los ciclos económicos. Como toda decisión de política económica, adoptar una moneda común implica beneficios y costos. Como se mostró más arriba, mientras mayor sea el grado de integración económica y financiera, mayores serán los beneficios, y más costoso renunciar a la posibilidad de adoptar una política monetaria nacional si los países enfrentan ciclos económicos diferentes. "Se desprende que un prerrequisito indispensable para el éxito de una unión monetaria es la concordancia entre las fluctuaciones de la actividad productiva de sus miembros" (De la Cuba y Winkelried, 2003: 4).

De su trabajo surge el postulado de que los miembros de una unión monetaria podrían cumplir de mejor forma ex-post los criterios de una AMO, dado que una moneda común lleva a una reducción de los costos de transacción y así se genera una mayor integración económica, un mayor comercio y, de allí, un grado de sincronización de ciclos económicos más alto. A la luz de este resultado se ha intentado verificar, realizando nuevos análisis con diversos datos y metodologías,

factores productivos y por ende mayor integración económica entre los países y mayores los beneficios de conformar una AMO. Ahora bien, la integración fiscal es deseable para poder minimizar la posibilidad de shocks asimétricos y ayuda a reducir los costos de perder el instrumento del tipo de cambio como mecanismo de ajuste. Ahora según Ingram (1973), la política fiscal debe ser independiente y tratar de dirigir la inversión hacia zonas deprimidas con el objetivo de estimular la demanda agregada y reducir el desempleo. Si esto no es posible, y la integración fiscal fuera reducida o nula, formar una AMO traería altos costos para los países que sufran shocks asimétricos. 
que aquellos países que presentan una relación comercial cercana tienden a tener ciclos económicos más fuertemente correlacionados.

Sin embargo, Krugman (1991) sostiene que una estrecha relación comercial lleva a que los países se especialicen y tengan estructuras productivas diferentes. Esto produciría una menor correlación de sus ciclos económicos y se verían afectados los países por shocks específicos de la industria. Este punto de vista es apoyado por Kalemli-Ozcan, Sorensen y Yosha (2001) que mostraron que una mayor integración en el mercado de capitales conduce a mejores ingresos y a asegurar una mayor especialización.

Frenkel y Rose (1998) toman en cuenta el efecto especialización que puede surgir por un incremento de la relación comercial pero sostienen que si los shocks de demanda (o shocks comunes) son los que predominan, o si prevalece el comercio intra-industrial para la mayoría del comercio, entonces los ciclos económicos se vuelven más similares entre los países cuando los países comercian mayor volumen.

En síntesis, existen teorías que llevan a realizar predicciones ambiguas acerca del rol de la integración económica y monetaria sobre la correlación de los ciclos económicos. La cuestión de si la especialización sectorial y la intensidad comercial afectan y en qué medida a la sincronización de ciclos, es empírica. Los resultados indican una relación fuerte y positiva: a mayor nivel de integración económica, mayor nivel de comercialización y mayor sincronización de ciclos.

Imbs (2004) encuentra a partir de un conjunto de datos para Europa y Estados Unidos, resultados similares a los de Frenkel y Rose (1998). El propósito de su trabajo es evaluar, mediante ecuaciones simultáneas, los determinantes de los ciclos económicos entre regiones y a lo largo del tiempo. Como determinantes toma al comercio, las finanzas y la especialización productiva. Los resultados a los que arriba muestran que el comercio y la apertura financiera tienen un efecto directo e indirecto en la sincronización de ciclos. Los patrones de especialización tienen un efecto medible pero débil sobre los ciclos económicos, más allá del comercio intra-industrial y la apertura al comercio de bienes y activos financieros. La especialización producida por el comercio no tiene virtualmente efecto alguno en la sincronización de ciclos económicos. El efecto del comercio inter-industrial es pequeño en comparación y consistente con los modelos existentes, en cambio, el efecto del comercio intra-industrial es fuerte y robusto en la sincronización de ciclos.

Traistaru (2004) y Artis (2003) también intentan verificar por un lado si existe un ciclo económico europeo y por el otro, cuáles son los canales de 
transmisión de sincronización o determinantes de los ciclos económicos en la zona de la unión europea y los nuevos socios. Los resultados del análisis empírico indican que la estructura económica similar y la intensidad bilateral comercial (usada como proxi de la integración económica) son positiva y significativamente asociados con la correlación de ciclos económicos. La relación entre los canales de sincronización y la coordinación de ciclos es encontrada endógena y sugiere, que en el largo plazo la convergencia de la estructura económica y el crecimiento del comercio son esperados. Este autor sugiere que a la luz de los resultados encontrados, la inclusión de nuevos países a la unión monetaria puede traerles efectos asimétricos. Los países tienen que evaluar costos y beneficios de renunciar a la política monetaria como herramienta de ajuste.

Traistaru-Siedschlag, junto con Tondl (2006), intentan investigar los patrones y determinantes de los co-movimientos de la actividad económica entre regiones en la Unión Europea y la Euro área. Trabajando con datos de 1989 al 2002 inclusive, realizan distintas estimaciones para analizar el impacto de la integración comercial regional, la especialización y volatilidad del tipo de cambio sobre las correlaciones de los ciclos de crecimiento regionales con la Euro área. Los resultados alcanzados indican que a mayor integración comercial con la Euro área, más fuerte, directo y positivo es el efecto sobre la sincronización de los ciclos de crecimiento regional con la Euro área. La especialización industrial y la volatilidad del tipo de cambio son fuentes de divergencia cíclica.

Por otro lado, algunos autores consideran que la hipótesis de la endogeneidad puede sostenerse para MERCOSUR dado que el proceso de integración de la región fue acompañado por un significativo aumento del comercio intra-industrial, más que en el bloque de la CAN.

Ciertos estudios han intentado testear esta hipótesis. Ahumada y MartirenaMantel (2001) emplean la metodología de Frenkel y Rose (1998) para evaluar y estimar conjuntamente los criterios AMO para el caso del MERCOSUR. Una de las conclusiones más llamativas de este trabajo es que las correlaciones entre la intensidad del comercio y la sincronía de los ciclos progresaron de negativas a positivas durante el período 1987-1999.

Otra evidencia que parece avalar esta hipótesis son los resultados obtenidos por Licandro Ferrando (1998). Aplicando el método de estimación de shocks de Bayoumi y Eichengreen (1994), el autor halla evidencia de que la mayor integración regional genera mayor similitud en los ciclos, aumentando la correlación de los shocks. Por otro lado, Fanelli et al. (2004) analizan los ciclos en 
el área del MERCOSUR, diferenciado shocks idiosincrásicos y shocks comunes. Ellos encuentran que los factores comunes que originan cambios en la inversión son relevantes para explicar co-movimientos en el producto y que la volatilidad es un factor relevante, el desarrollo financiero importa tanto para la volatilidad como para la dinámica del producto y los precios.

\section{DISCUSION}

Hasta el momento se han identificado tres fuentes de sincronización de ciclos económicos. Por un lado tenemos la apertura o integración económica, la cual conduce a incrementar el comercio y los flujos de inversión y la integración financiera entre los socios. Por el otro, siguiendo a Kenen (1969), cuánta mayor similitud haya entre las estructuras económicas de los países, mayor será el nivel de correlación de los ciclos, y menor el costo de renunciar a la posibilidad de adoptar un política monetaria autónoma como herramienta de ajuste ante shocks de demanda u oferta externos. La tercera fuente de sincronización de ciclos es la vinculación política entre los países, es decir, la coordinación de las políticas macroeconómicas. De acuerdo con la teoría de los ciclos económicos, dicha coordinación puede tener el efecto de producir menos variaciones cíclicas entre los miembros de la unión si dicha política es considerada en sí misma como fuente de fluctuación del ciclo económico.

Con respecto a establecer una AMO en Latinoamérica, De la Cuba y Winkelried (2004) arriban a la conclusión de que no se recomienda una moneda única debido a la falta de coordinación de los ciclos económicos. Pero que los ciclos observados actualmente no necesariamente son los mismos que enfrentarían sus economías en etapas más avanzadas de integración. Entonces, es necesario que los países armonicen sus políticas internas.

La evidencia empírica indica que a pesar de los esfuerzos consecuentes de integración entre las economías de Latinoamérica, la reducción de diferencias para tender a la convergencia no ha sido suficiente. Madariaga, Montout y Ollivaud (2003) encuentran resultados que respaldan convergencia para los países miembros del NAFTA, pero no así para el MERCOSUR. De igual modo, González, Dabús y Monterubbianesi (2009), Dobson y Ramlogan (2002) y Dabús y Zinni (2005), tampoco encuentran evidencia sobre convergencia para los países de América Latina. Aunque existan indicios de divergencia en la región, algunos autores sugieren la posibilidad de formación de clubes de convergencia, aunque aún no existe un consenso al respecto. 
Los resultados empíricos observados por autores como De la Cuba y Winkelried (2004) y Escaith (2003) no son muy alentadores. Si bien en el trabajo muestran que debido a que el aumento de bienestar a que da lugar la cooperación es endógeno, incluso en las primeras etapas cuando los socios se ven tentados a romper con el acuerdo de coordinación, en algún momento la misma se tornará estable. A nivel regional las condiciones observadas son más promisorias que para toda América Latina, pero aún distan mucho de ser autosostenidas.

Escaith (2003) y Fanelli et. al. (2004) sugieren que dado que la coordinación es inestable, se necesita un marco institucional formal para iniciar y coordinar el proceso. Además habría que aplicar políticas más tradicionales de integración económica, porque antes de la unión monetaria hay que avanzar en la integración comercial e ir adoptando una política comercial externa común para los no miembros, llevar a cabo la unificación de las instituciones económicas y el desarrollo de algunas instituciones supranacionales cuyas decisiones se aplican a todos los miembros y la eliminación de las barreras a los movimientos de los factores productivos.

En síntesis, un país al momento de tomar la decisión de unirse o formar parte de una unión monetaria debe analizar los beneficios y costos bajo ciertos criterios, sin olvidarse que los mismos tienen una relación entre sí endógena, por lo tanto, aquellas condiciones que parecen no cumplir es posible que las cumpla luego de haber ingresado a la unión. Cuanta mayor intensidad comercial, similitud de estructuras económicas y coordinación de políticas se observe entre los miembros de la unión, mayor sincronización de ciclos económicos, y por lo tanto, mayor aumento de bienestar para las sociedades de los países socios.

Si bien es difícil identificar un ciclo europeo homogéneo, el grado de simetría que presentan los ciclos de los países miembros de la Unión Europea es mayor al observado para los países de América Latina. Siguiendo a De la Cuba y Winkelried (2004), en el corto plazo es apresurado hablar de una unión monetaria latinoamericana pero sus hallazgos sugieren evaluar con mayor detenimiento la posibilidad de llevar a cabo dicha unión a nivel de subgrupos de países o regiones. 


\section{REFERENCIAS BIBLIOGRÁFICAS}

Ahumada, H. \& Martirena-Mantel, A. M. (2001). Towards a potencial monetary union in Latin America: testing the endogeneity of the criteria for Mercosur. Anales. Buenos Aires: Asociación Argentina de Economía Política Recuperado de http://www.aaep.org.ar/anales/works/works2001/ ahumada_martirena-mantel.pdf

Allen, P.R. (1982). Increased wage or productivity differentials in a monetary union. In M.T. Sumner, \& G. Zis (Eds). European Monetary Union: Progress and Prospects. (pp. 195-215) London: Macmillan.

Allen, P. R. (1983). Policies to Correct Cyclical Imbalance Within a Monetary Union. Journal of Common Market Studies, 21 (3), 313-328.

Artis, M. J. (2003). Is there a European Business Cycle? CESifo, Working Paper Series No. 1053. Recuperado de file://C:/Documents\%20and\%20Settings/ Eduth2/Mis\%20documentos/Descargas/cesifo1_wp1053\%20(1).pdf

Artis, M. J., \& Zhang W. (1997). The international business cycle and the ERM: is there a European Cycle?. International Journal of Finance \& Economics, $2(1), 1-16$.

Balassa, B. (1964). Teoría de la integración económica. México: UTEHA

Bayoumi, T., \& Eichengreen, B. (1994). One Money or Many? Analysing the prospects for Monetary Unification in Various Parts of the World. Department of Economics Princeton University, Princeton Studies in International Economics No. 76. Recuperado de https://www.princeton. edu/ ies/IES_Studies/S76.pdf

Commission of the European Communities (1992). Treaty on European Union. Luxembourg: European Communities.

Dabús, C.,\& Zinni, B. (2005). No convergencia en América Latina. Trabajo publicado en los Anales de la XL Reunión de la Asociación Argentina de Economía Política, UNLP. La Plata: AAEP Recuperado de http://www. aaep.org.ar/anales/works/works2005/dabus_zinni.pdf

De la Cuba, M.,\& Winkelried, D. (2004). ¿Una moneda común?: Evidencias para América Latina. Integración \& comercio, 20, 203-229. Recuperado de http://www10.iadb.org/intal/intalcdi/integracion_comercio/e_INTAL_ IYC_20_2004_PremioINTAL.pdf

Dobson, S., \& Ramlogan, C. (2002). Economic growth and convergence in Latin America. Journal of Development Studies, 38 (6), 83-104.

Escaith, H. (2003). La integración regional y la coordinación macroeconómica en América Latina. Revista de la CEPAL, 82, 55-74.

Fanelli, J. M., González Rozada, M. \& Kaifman S. (2004). Coordinación de políticas macroeconómicas en el Mercosur. Madrid: Siglo XXI. 
Fleming, J. M. (1971). On exchange rate unification. The economic Journal, vol. 81, 467-488.

Frenkel, J., \& Rose A.K. (1998). The Endogeneity of the Optimum Currency Area Criteria. Economic Journal, 108 (450), 1009-1025.

González, G. H., Dabús, C., \& Monterubbianesi, P. (2009). Convergencia en economías semi-industrializadas: nueva evidencia de América Latina y Caribe. Trabajo presentado en la XLIV Reunión Anual de la Asociación Argentina de Economía Política. Mendoza: AAEP.

Recuperado de http://www.aaep.org.ar/anales/works/works2009/gonzalez.pdf

Imbs, J. (2004). Trade, finance, specialization and synchronization. Review of Economics and Statistics, 86 (3).

Ingram, J. C. (1973). The Case of European Monetary Integration. Essays in International Finance No. 98. Princeton, New Jersey: International Finance Section, Princeton University.

Kalemli-Ozcan, S., Sørensen, B. E., \& Yosha, O. (2001). Economic integration, industrial specialization, and the asymmetry of macroeconomic fluctuations. Journal of International Economics, 55 (1), 107-137.

Kenen, P. (1969) The theory of optimum currency areas: an eclectic view. In Mundell, R., \& Swoboba, K. (Eds.). Monetary problems of the international economy. University of Chicago Press, 41-60.

Krugman, P. R. (1991). Geography and trade. Belgium and London: Leuven University Press and The MITT Press.

Krugman, P. R \& Obstfeld, M. (2003). International Economics, theory and Policy (6th ed.). Boston: Addison Wesley.

Licandro Ferrando, G. (1998). Is MERCOSUR an Optimal Currency Area? A Shock Correlation Perspective. Trabajo presentado en el Congreso ETC, 12 de junio de 1998, Versión preliminar. Banco Central del Uruguay, Montevideo.

MacDougall, G. D. A. (1975). Discussion Paper. In Report of the Study Group on Economic and Monetary Union in 1980, Annex II. Brussels: Commission of The European Communities, pp. 97-103.

Madariaga, N., Montout, S., \& Ollivaud, P. (2003). Regional convergence, trade liberalization and aglomeration of activities: an analysis of NAFTA and Mercosur cases. Maison de Sciences Economiques, Université Paris.

Manchón, F. (2004). El argumento del área monetaria óptima y la Unión Monetaria de América del Norte. En V. M. Soria \& G. Vidal (Coords.). México en la región de América del Norte: problemas y perspectivas, (pp. 201-229).

Martínez Romera, J. P. (2013). Teoría de las Áreas Monetarias Óptimas: Una revisión de la literatura desde una perspectiva europea. Universidad Autónoma de Madrid, Economic Analysis Working Papers Series No. 4. 
Recuperado de http:/www.uam.es/departamentos/economicas/analecon/ especifica/mimeo/wp20134.pdf

Martirena- Mantel, A. (1997). Reflexiones sobre Uniones Monetarias: Pensando al Mercosur desde el caso europeo. Buenos Aires: Anales de la Academia Nacional de Ciencias Económicas.

McKinnon, R. (1963). Optimum Currency Area. American Economic Review, 53 (4), 717- 725.

Meade, J. E. (1951). The Balance of Payments. Londres: Oxford University Press. Mundell, R. (1961). A theory of Optimum Currency Area. American Economic Review, 51 (4), 567- 665.

Robson, P. (1998). The economics of international integration (4th ed.). London: Routledge.

Scitovsky, T. (1958). Economic Theory and Western European Integration. Stanford, California: Stanford University Press.

Tondl, G., \& Traistaru-Siedschlag I. (2006). Regional growth cycle convergence in the European Union. University of Economics and Business Administration Vienna, Working Paper No. 71.

Traistaru, I. (2004). Transmission Channels of Business Cycles Synchronization in an Enlarged EMU. Germany: University of Bonn, Working Paper B18. Recuperado de https://www.zei.uni-bonn.de/dateien/working-papaer/B0418.pdf

Tugores Ques, J. (1997). Integración Comercial. En J. Tugores Ques. Economía internacional e integración económica (3a. ed.), (pp. 149-181). México: McGraw-Hill.

(C) 2015 por los autores; licencia otorgada a la Revista Estudios Económicos. Este artículo es de acceso abierto y distribuido bajo los términos y condiciones de una licencia Atribución-No Comercial 3.0 Unported (CC BY-NC 3.0) de Creative Commons. Para ver una copia de esta licencia, visite http://creativecommons.org/ licenses/by-nc/3.0/ 